\section{Variability of cadmium accumu- lation in cephalopods (Octopus vulgaris, Sepia officinalis, Loligo vulgaris and Todarodes sagittatus) collected in Sardinia in 2008-2012}

\author{
Pierluigi Piras, ${ }^{1}$ Giannina Chessa, ${ }^{2}$ \\ Maurizio Cossu, ${ }^{2}$ Federica Rubattu, ${ }^{2}$ \\ Gianuario Fiori $^{2}$
}

'Scuola di Dottorato di Ricerca in Produzione e Sicurezza degli Alimenti di Origine Animale, Dipartimento di Medicina Veterinaria, Università degli Studi di Sassari; '2stituto Zooprofilattico Sperimentale della Sardegna G. Pegreffi, Sassari, Italy

\section{Abstract \\ Cephalopods are known to accumulate cad-} mium and play an important role in its biomagnification. They are an essential link in marine trophic chain and represent an important case in studies on cadmium transfer to man through the food chain. Since cadmium concentration widely varies in different tissues of the cephalopods - mainly accumulating in the hepatopancreas - evisceration represents a recommended preliminary step to reduce cadmium intake in view of cephalopods consumption; yet, the residual concentration in the edible part may still be a risk for public health. This study is intended to assess cadmium levels variability in the muscles of Cephalopoda, considering the different feeding habitats and marine trophic webs. In compliance with EU regulation, a survey on cadmium and other heavy metal levels in various sea food, including cephalopods, was conducted by the Istituto Zooprofilattico of Sardinia in co-operation with local health authorities. During a five-year survey (20082012), 90 samples were collected from the following species: commons octopus (Octopus vulgaris), cuttlefish (Sepia officinalis), European squid (Loligo vulgaris), and flying squid (Todarodes sagittatus), located in different coastal areas and representatives of either benthic or nektonic habitats. Determination of cadmium levels was carried out according to Regulations (EC) No. 882/2004, No. 1881/2006 and No. 333/2007. Analysis of the edible portion (muscle) of fresh homogenised samples was carried out by inductively coupled plasma mass spectrometry (ICP-MS). The results showed a highly skewed distribution of data. No statistically significant differences were observed among four distributions of the natural logs of cadmium levels in the species considered.

\section{Introduzione}

Il cadmio (Cd) è un metallo presente a basse concentrazioni nella crosta terrestre, associato con i minerali di zinco, piombo e rame. Come ha recentemente rimarcato anche la World Health Organization (WHO, 2010), la sua concentrazione può aumentare attraverso attività antropiche, in particolare tramite le attività estrattive, sia funzionanti che dismesse, essendo il Cd tipicamente reperito come sottoprodotto minerario e/o legato allo smaltimento di code minerarie, oltre che proveniente dall'industria dei combustibili fossili 0 dai rifiuti industriali. Una volta nei suoli, il Cd può diventare mobile ed essere assorbito dalla vegetazione e/o venire disperso negli ambienti acquatici. Si tratta, come è noto, di un elemento chimico non essenziale, potenzialmente tossico per gli organismi animali. I cefalopodi, tuttavia, come altri molluschi acquatici, dispongono di meccanismi di difesa verso tale elemento e verso altri metalli pesanti. Nella fattispecie, i cefalopodi ricorrono al sequestro dei metalli pesanti: l'isolamento nella ghiandola digestiva 0 epatopancreas di alcuni metalli pesanti bio-tossicologicamente importanti come il Cd rappresenta infatti una tendenza generale di tale gruppo zoologico (Nessim e Riad, 2003; Bernardi et al., 2004; Raimundo et al., 2004, 2005). Quando il Cd raggiunge tale organo, si lega a molecole del citosol, in particolare quelle aventi gruppi sulfidrilici, denominate genericamente metallotioneine (Bustamante et al., 2002a; Raimundo et al., 2010a), diventando metabolicamente indisponibile. Per la detossificazione a lungo termine, il Cd può inoltre essere depositato nei lisoso$\mathrm{mi}$, come precipitato in granuli insolubili di minerali di solfuro o fosfato, ed essere quindi definitivamente rimosso dal pool come ione biologicamente attivo (Bustamante et al., 2006; Raimundo et al., 2010b). I cefalopodi rappresentano a loro volta una fonte importante di $\mathrm{Cd}$ per i relativi predatori, agendo come importanti vettori nel processo di biomagnificazione (Bustamante et al., 1998; Raimundo et al., 2008; Chouvelon et al., 2011). Proprio il ruolo che essi possono svolgere nella distribuzione del Cd ai livelli trofici superiori ha motivato gli studi sull'accumulo di Cd nei cefalopodi in condizioni di esposizione controllata a breve termine e/o a mimare l'esposizione cronica (Bustamante et al., 2002b). Le concentrazioni di Cd possono infatti variare tra i diversi tessuti e i diversi stadi di vita (Storelli et al., 1999). Tuttavia il $\mathrm{Cd}$, come si è detto, è accumulato nei visceri e soprattutto nell'epatopancreas (Storelli et al., 2005; Miramand et al., 2006; Manso et al., 2007; Raimundo et al., 2008; Bonerba et al., 2009; Pereira et al., 2009) e, pertanto, l'eviscerazione rappresenta un'operazione preliminare raccomandata ai fini della
Correspondence: Pierluigi Piras, Scuola di Dottorato di Ricerca in Produzione e Sicurezza degli Alimenti di Origine Animale, Dipartimento di Medicina Veterinaria, Università degli Studi di Sassari, via Vienna 2, 07100 Sassari, Italy. Tel. +39.339.2056525 - Fax: +39.079.229869. E-mail: pirasp@tiscali.it

Key words: Cadmium, Cephalopods, Bioaccumulation, Food safety.

Acknowledgements: the authors are thankful to Antonino Bella, researcher at the Istituto Superiore di Sanità (Roma), whose help was fundamental in statistical analysis.

Received for publication: 15 January 2013.

Revision received: 20 March 2013.

Accepted for publication: 24 March 2013.

This work is licensed under a Creative Commons Attribution 3.0 License (by-nc 3.0).

(C) Copyright P. Piras et al., 2013

Licensee PAGEPress, Italy

Italian Journal of Food Safety 2013; 2:e24

doi:10.4081/ijfs.2013.e24

significativa riduzione dell'assunzione di $\mathrm{Cd}$ conseguente al loro consumo. I consumatori sono infatti esposti a varie dosi di Cd a seconda della via di esposizione; per la popolazione generale (esclusi in particolare i fumatori) il percorso dominante è però la via alimentare (Storelli et al., 2006, 2010). L'esposizione alimentare umana è stata affrontata anche recentemente, sia in riferimento alla popolazione italiana (Pastorelli et al., 2011) che a quella dell'Unione Europea (EFSA, 2012). L'esposizione al Cd attraverso prodotti alimentari è infatti di costante preoccupazione per la salute pubblica (EFSA, 2009; WHO, 2010), poiché quella a lungo termine dà luogo ad accumulo nell'organismo e può provocare effetti negativi di varia natura, anche gravi. Il $\mathrm{Cd}$ ha infatti una lunga emivita biologica (stimata in circa 15-30 anni), cui consegue la grande capacità di accumularsi nell'organismo. Organi bersaglio critici dell'esposizione a basse concentrazioni di Cd sono soprattutto fegato e reni, nei quali si deposita il $30-60 \%$ dell'elemento. Dal punto di vista delle proprietà cancerogene, inoltre, l'International Agency for Research on Cancer (IARC) ha classificato il $\mathrm{Cd}$ e i suoi composti come cancerogeni umani di classe 1. Sulla base dell'analisi del rischio, il Joint FAO/WHO Expert Committee on Food Additives (JECFA) di esperti del Food and Agriculture Organization/World Health Organization FAO/WHO aveva quindi stabilito un tolerable weekly intake (TWI) provvisorio di $7 \mu \mathrm{g} / \mathrm{kg}$ di peso corporeo, a seguito del quale molte agenzie di regolamentazione in tutto il mondo fornirono però evidenze per un suo rie- 
same, considerate le preoccupazioni per i margini di sicurezza dello standard proposto. Così ha fatto il Panel europeo di esperti per la sicurezza alimentare sui contaminanti nella catena alimentare, che ha raccomandato (EFSA, 2009) e ribadito (EFSA, 2011) l'orientamento per un ridotto TWI, pari a $2,5 \mu \mathrm{gg} / \mathrm{kg}$ di peso corporeo.

Col presente contributo si è voluto valutare la variabilità dell'accumulo di Cd nelle parti edibili (muscolo) in quattro specie di cefalopodi, comunemente presenti sui nostri mercati, oltre che provenienti da diverse zone della costa e rappresentanti diversi ambienti, sia nectonici che bentonici. Tale classe dei molluschi sta peraltro attirando una sempre maggior attenzione da parte delle discipline biologiche applicate al mare, assumendo una crescente importanza per la pesca europea (Pierce et al., 2010). In particolare, il polpo Octopus vulgaris (famiglia Octopodidae) è una specie tipicamente bentonica e costiera (neri-tica), di acque basse 0 poco profonde (quasi mai sotto i $100 \mathrm{~m}$ ), che vive una vita solitaria negli anfratti rocciosi del fondo e tra le praterie di fanerogame marine; la seppia Sepia officinalis (famiglia Sepiidae) è invece una specie necto-bentonica (demersale) della zona litorale e relativa scarpata continentale, che frequenta habitat diversi, come roccia, sabbia, fango, coralli e alghe, in prossimità della costa e fino a $150 \mathrm{~m}$ di profondità, dove si trova con più abbondanza; il calamaro Loligo vulgaris (famiglia Loliginidae) è anch'essa una specie costiera, semi-pelagica, con una distribuzione più 0 meno estesa, comunque generalmente mai al di sotto della piattaforma continentale, e compie migrazioni stagionali tra basse batimetrie e 250 m di profondità; il totano Todarodes sagittatus (famiglia Ommastrephidae) è, infine, una specie pelagica e semi-demersale, che vive tra la superficie e $800 \mathrm{~m}$ di profondità e compie significative migrazioni sia stagionali che diurne verticali, stando vicino al fondo durante il giorno. Tutte e quattro le specie sono carnivore e si nutrono principalmente di crostacei, piccoli pesci e molluschi, compresi i conspecifici. Il processo di bioaccumulo di metalli pesanti è stato infatti descritto come passibile di variazione a seconda delle specificità degli organismi, come l'età/dimensione, sesso ed ecologia, oltre che secondo la biodisponibilità relativa del metallo nella dieta e nell'acqua di mare (Seixas et al., 2005; Pierce et al., 2008; Raimundo e Vale, 2008).

Oggetto dell'indagine è l'analisi comparativa delle concentrazioni di Cd tra le quattro specie di cefalopodi. L'appartenenza a nicchie ecologiche diverse ha rappresentato il presupposto per la valutazione dell'eventuale influenza della specie e, indirettamente, dell'ambiente sulla capacità di tali organismi di bioaccumulare Cd nella parte edibile. In altri termini, tenuto conto che la normativa comunitaria esclude i visceri relativamente alla determinazione del $\mathrm{Cd}$, lo scopo del presente studio è stato quello di indagare la variabilità intra- ed inter-specifica della sua distribuzione nella sola parte edibile e, quindi, valutare in che misura tale variabilità potesse rappresentare un fattore di rischio per i consumatori.

\section{Case Report}

Nel quinquennio 2008-2012 sono stati sottoposti ad analisi per la ricerca del Cd da parte dell'Istituto Zooprofilattico Sperimentale (IZS) della Sardegna 90 campioni di cefalopodi prelevati dai Servizi Veterinari delle ASL della Regione Sardegna, appartenenti alle specie polpo (Octopus vulgaris), seppia (Sepia officinalis), calamaro (Loligo vulgaris) e totano (Todarodes sagittatus). Il campionamento e la determinazione dei livelli di Cd nei cefalopodi sono stati svolti in conformità ai Regolamenti (CE) n. 882/2004 (Commissione Europea, 2004) e n. 333/2007 (Commissione Europea, 2007). Il Cd è stato determinato sull'omogenato della parte muscolare, escludendo l'epatopancreas, così come previsto dal Regolamento (CE) n. 1881/2006 (Commissione Europea, 2006) al relativo allegato (punto 3.2.11 del testo consolidato) che, per i cefalopodi, stabilisce tenori massimi da determinarsi nei prodotti senza visceri. Le concentrazioni di Cd sono state determinate in applicazione al metodo EPA 6020A (EPA, 2007) per l'analisi strumentale in associazione al metodo EPA 3052 (EPA, 1996) per gli aspetti che riguardano il trattamento del campione. Una porzione (1 gr) del campione omogenato è

stata sottoposta a degradazione ossidativa mediante mineralizzazione acida, a temperatura e pressione controllata, in forno a microonde. La soluzione risultante, opportunamente diluita, è stata analizzata tramite tecnica inductively coupled plasma mass spectrometry (ICP-MS), strumentazione Agilent 7500CE (Agilent Technologies, Cernusco Sul Naviglio, Italia). Le concentrazioni sono state determinate per interpolazione del segnale di lettura con curve di taratura utilizzando il ${ }^{111} \mathrm{Cd}$, quale isotopo di quantificazione, e il ${ }^{115} \mathrm{In}$, quale standard interno. Le sequenze analitiche hanno previsto, oltre all'analisi dei campioni in esame, la lettura di soluzioni di controllo relative a bianco-reattivi e matrici certificate CRM 278 e CRM DOLT-2. La procedura analitica utilizzata è stata validata in conformità con la norma UNI EN ISO 17025/2005 (ISO, 2005) e presenta un limit of quantification (LoQ) pari a $0,010 \mathrm{mg} / \mathrm{kg}$. Nell'analisi dei dati, non disponendo di una numerosità campionaria sufficiente ad indagare per variabili intraspecifiche (come la gamma di dimensioni e taglia in relazione all'età, e/o per provenienza geografica), ci si è concentrati a comparare solo la variabilità delle concentrazioni del metallo tra le quattro specie. Relativamente ai test statistici, l'analisi della varianza [tramite ANalysis of Variance (ANOVA)] delle concentrazioni di Cd è stata svolta su log-trasformate naturali, dopo aver comunque saggiato l'ipotesi della distribuzione normale, ricorrendo al test di Shapiro-Wilk e, per l'ipotesi dell'uguaglianza della varianza (o della omoschedasticità), ricorrendo al test di Bartlett. L'indagine, svolta con lo scopo di verificare se e in quale misura l'accumulo di Cd nei muscoli potesse significativamente dif-

Tabella 1. Range, media ( \pm standard deviation) e mediana delle concentrazioni di cadmio (mg/kg di peso fresco) su cefalopodi senza visceri.

\begin{tabular}{llccc} 
Specie & N & Range & Media \pm SD & Mediana \\
Octopus vulgaris & 21 & $0,001-0,715$ & $0,148 \pm 0,211$ & 0,031 \\
Sepia officinalis & 23 & $0,001-1,775$ & $0,257 \pm 0,493$ & 0,017 \\
\hline Loligo vulgaris & 31 & $0,002-2,700$ & $0,249 \pm 0,552$ & 0,054 \\
Todarodes sagittatus & 15 & $0,007-5,189$ & $0,675 \pm 1,476$ & 0,052 \\
\hline
\end{tabular}

SD, standard deviation.

Tabella 2. Media ( \pm standard error) e mediana delle distribuzioni log-naturali delle concentrazioni di cadmio (mg/kg di peso fresco) su cefalopodi senza visceri.

\begin{tabular}{lcccc} 
Specie & $\mathbf{N}$ & Media in log-nat & \pm SE & Mediana in log-nat \\
Octopus vulgaris & 21 & 3,611745 & $\pm 0,491848$ & 3,473768 \\
Sepia officinalis & 23 & 3,368072 & $\pm 0,458345$ & 4,074542 \\
\hline Loligo vulgaris & 31 & 2,980759 & $\pm 0,337674$ & 2,918771 \\
Todarodes sagittatus & 15 & 2,229905 & $\pm 0,479660$ & 2,956512 \\
\hline Totale & 90 & 3,101827 & $\pm 0,218156$ & 3,026670
\end{tabular}

$\mathrm{SE}$, standard error. 
ferire tra le quattro specie di cefalopodi più rappresentative del pescato mediterraneo, ha consentito una prima analisi delle distribuzioni, con i risultati riportati nella Tabella 1 . Nel complesso, il limite di tolleranza stabilito dalla normativa comunitaria, pari a $1,0 \mathrm{mg} / \mathrm{kg} \mathrm{di}$ peso fresco nel prodotto senza visceri, è stato superato in 7 campioni su 90 (7,8\%). Per confrontare i livelli medi di $\mathrm{Cd}$ nelle diverse specie si è ipotizzato di ricorrere, in prima battuta, ad un test parametrico, ovviamente dopo verifica delle precondizioni della statistica parametrica (ovvero sulla normalità delle distribuzioni e sulla loro omoschedasticità), e solo in caso contrario si è considerata l'alternativa di ricorrere residualmente ad un test non parametrico. La variabile del livello di Cd riscontrato nelle diverse specie di cefalopodi presentava da subito una distribuzione fortemente asimmetrica a destra (Figura 1). Inoltre, anche nell'eventualità di calcolare il test della normalità di Shapiro-Wilk, questo risultava essere senza dubbio significativo $(\mathrm{P}<0,0001)$, avendo conferma che la variabile Cd nel muscolo dei cefalopodi non risultava normalmente distribuita. Con tali premesse, si è dovuto perciò cautelativamente fare ricorso a un test non parametrico (nel caso specifico, quello di Kruskal-Wallis, noto anche come equality of populations rank test) che ha restituito un chi-quadro di 3,7810 con 3 gradi di libertà, quindi non significativo $(\mathrm{P}=0,2863)$. Ciò ha portato ad una prima constatazione che la differenza tra i livelli medi di Cd nelle quattro specie di cefalopodi non risultasse statisticamente significativa ricorrendo ad un test non parametrico. Volendo però utilizzare anche un test parametrico (che, sotto il profilo statistico sarebbe risultato ovviamente più robusto), si è voluto fare un tentativo di normalizzazione delle distribuzioni attraverso la trasformazione logaritmica. In questo caso, la distribuzione era diventata piuttosto simmetrica (Figura 2) e il test della normalità di Shapiro-Wilk era risultato non significativo anche in questo caso $(\mathrm{P}=0,2242)$, dando così evidenza del fatto che la trasformazione logaritmica dei livelli di Cd avesse veramente normalizzato la relativa distribuzione. Non è restato perciò che verificare l'altra precondizione della statistica parametrica, ovvero la omoschedasticità delle quattro distribuzioni log-naturali, attraverso l'utilizzo del test di Bartlett. Quest'ultimo è risultato non significativo $(\mathrm{P}=0,739)$, tanto che le varianze sono da ritenersi significativamente omogenee e la condizione di omoschedasticità quindi verificata. Per confrontare le medie log-naturali dei quattro gruppi di cefalopodi si è quindi passati all'utilizzo del test ANOVA, con i valori riferiti ai quattro gruppi riepilogati nella Tabella 2 e con risultato non significativo $(\mathrm{P}=0,2205)$. Anche il test parametrico ha confermato che la differenza tra i livelli medi di Cd nelle quattro specie di cefalopodi non era statisticamente significativa.

\section{Discussione}

I cefalopodi sono in grado di accumulare $\mathrm{Cd}$ in diversi tessuti, con livelli notoriamente alti nell'epatopancreas e relativamente molto più bassi nel muscolo. Per quanto riguarda la variabilità dell'accumulo in tale tessuto, dalla presente indagine si possono trarre le seguenti conclusioni: i) la distribuzione delle frequenze di concentrazione del Cd nel muscolo delle specie di cefa- lopodi esaminati risulta nel complesso essere di tipo log-normale, come lo sono le specifiche distribuzioni nei quattro gruppi; ii) assumendo quindi l'ipotesi di base dell'analisi della varianza (ANOVA) testata nello studio, ovvero la possibile scomposizione della varianza nelle sue due componenti, quella interna ai gruppi (o within) e quella tra $i$ gruppi (o between), si è potuto evidenziare come la prima componente risultasse relativamente elevata rispetto alla seconda, in misura tale che, con alta significatività statistica, la differenza tra i quattro gruppi fosse praticamente attribuibile solo alla variabilità interna. In altre parole: nel confronto in esame, la varianza between non contribuirebbe significativa-

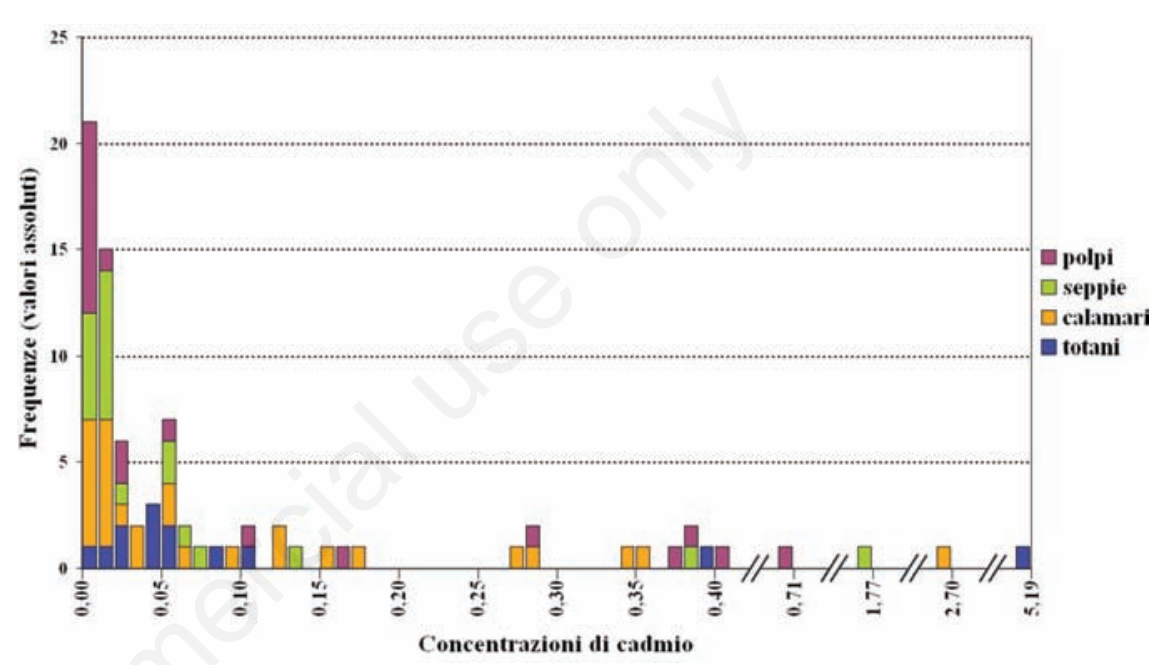

Figura 1. Distribuzione delle concentrazioni di cadmio $(\mathrm{mg} / \mathrm{kg}$ di peso fresco su cefalopodi senza visceri) espresse come valori assoluti in istogramma a barre sovrapposte. L'asse delle concentrazioni si è dovuto ripetutamente spezzare a destra a causa della lunga coda delle distribuzioni, volendo comunque evidenziare i valori estremi di range per le quattro specie.

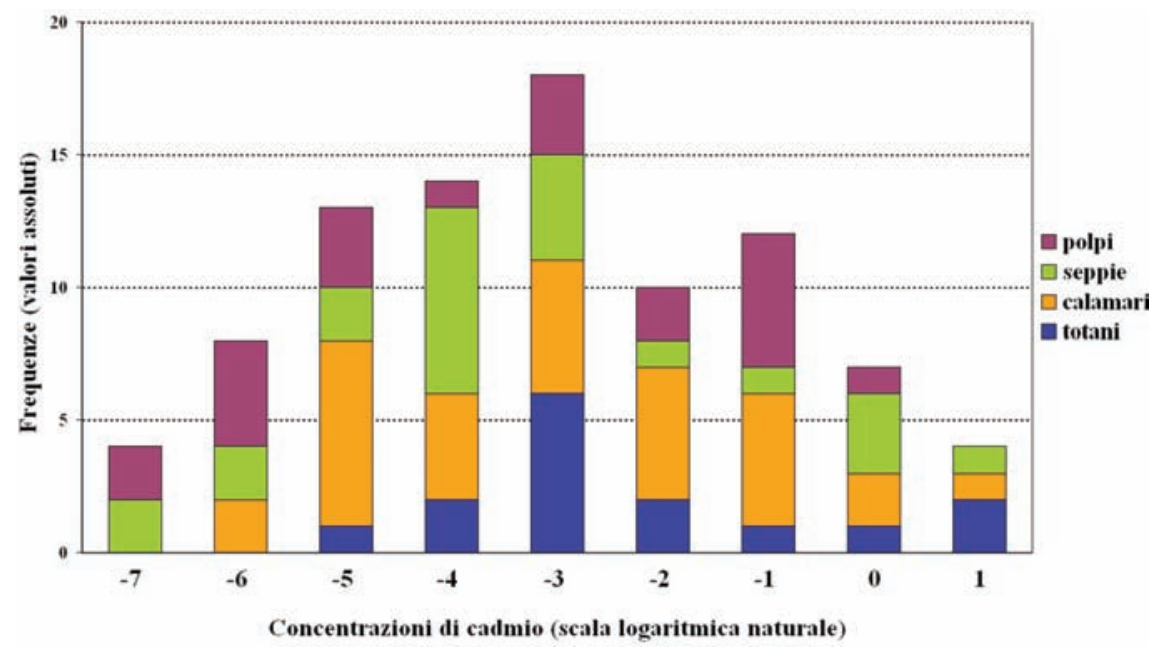

Figura 2. Distribuzione delle concentrazioni di cadmio $(\mathrm{mg} / \mathrm{kg}$ di peso fresco su cefalopodi senza visceri) espresse come valori log-naturali in istogramma a barre sovrapposte. 
mente alla varianza totale ed il fenomeno quindi non sarebbe legato a caratteristiche proprie di ciascuna delle quattro specie di cefalopodi.

\section{Conclusioni}

Dal momento che con la presente indagine non è stata evidenziata una variabilità significativamente diversa nei livelli di $\mathrm{Cd}$ nel muscolo tra i quattro gruppi, lo scenario più probabile che differenzierebbe ontologicamente le quattro specie sarebbe soprattutto legato alle condizioni di partizionamento del $\mathrm{Cd}$ nell'epatopancreas. I risultati del presente studio osservazionale sulla variabilità della distribuzione del $\mathrm{Cd}$ nel muscolo dei cefalopodi, è pertanto solo apparentemente contrastante con i risultati di altri studi svolti in condizioni sperimentali, dove sono state rilevate differenze nei meccanismi di accumulo del $\mathrm{Cd}$, in particolare nell'epatopancreas, tra le diverse specie di cefalopodi. Come riferito da diversi autori (Storelli et al., 1999; Bustamante et al., 2002a, 2002b; Nessim e Riad, 2003; Raimundo et al., 2004, 2010a; Storelli et al., 2005, 2006, 2010; Bonerba et al., 2009; Pereira et al., 2009), i modelli differenti di partizionamento del $\mathrm{Cd}$ all'interno dei tessuti, in particolare dell'epatopancreas, benché sottintendano alcune significative differenze tra le specie, non determinerebbero infatti carichi di Cd molto dissimili a livello muscolare (Raimundo et al., 2005; Manso et al., 2007; Chouvelon et al., 2011). Pertanto, ciò non deve essere preso in alcun modo come prova del fatto che il partizionamento ed il metabolismo del Cd siano identici in tutte le specie. Le ragioni delle differenze interspecifiche di bioaccumulazione nei visceri, epatopancreas in particolare, riferite in bibliografia (e, per la specificità d'organo, biologicamente spiegate), si ritiene possano quindi plausibilmente essere compatibili con la significativa assenza di differenze osservata invece nei muscoli, data la fisiologia muscolare presumibilmente simile nelle specie considerate. A tale proposito, si ritengono però necessari ulteriori studi ed un prosieguo dell'indagine per confermare il fenomeno su scala più ampia. Al momento, il presente studio costituisce un contributo pratico per coloro che valutano sul campo il rischio $\mathrm{Cd}$ nei cefalopodi destinati al consumo umano - da consumarsi comunque senza visceri. Nella fattispecie, lo studio qui descritto considera tale classe di molluschi tendenzialmente omogenea relativamente al rischio $\mathrm{Cd}$ ad essa associato, come d'altronde dispone la stessa normativa comunitaria che stabilisce a tutt'oggi un tenore massimo unico per la categoria generica dei cefalopodi.

\section{Bibliografia}

Bernardi C, Trezzi I, Renon P, Cattaneo P, Malandra R, 2004. Determinazione di piombo e cadmio in molluschi cefalopodi del Mediterraneo. Ind Aliment-Italy 43:1006-8.

Bonerba E, de Candia G, Ceci E, 2009. Presenza di cadmio in Octopus vulgaris: valutazione del rischio per la salute pubblica. Rivista dell'AIVI 6:73-6.

Bustamante P, Bertrand M, Boucaud-Camou E, Miramand $\mathrm{P}, 2006$. Subcellular distribution of $\mathrm{Ag}, \mathrm{Cd}, \mathrm{Co}, \mathrm{Cu}, \mathrm{Fe}, \mathrm{Mn}, \mathrm{Pb}$, and $\mathrm{Zn}$ in the digestive gland of the common cuttlefish Sepia officinalis. J Shellfish Res 25:98793.

Bustamante P, Caurant F, Fowler SW, Miramand P, 1998. Cephalopods as a key of the transfer of cadmium to top marine predators. Sci Total Environ 220:71-80.

Bustamante P, Cosson RP, Gallien I, Caurant F, Miramand P, 2002a. Cadmium detoxification processes in the digestive gland of cephalopods in relation to accumulated cadmium concentrations. Mar Environ Res 53:227-41.

Bustamante P, Teyssié JL, Fowler SW, Cotret 0, Danis B, Miramand P, Warnau M, 2002b. Biokinetics of cadmium and zinc accumulation and depuration at different stages in the life cycle of the cuttlefish Sepia officinalis. Mar Ecol-Prog Ser 231:167-77.

Chouvelon T, Spitz J, Cherel Y, Caurant F, Sirmel R, Mèndez-Fernandez $P$, Bustamante P, 2011. Inter-specific and ontogenic differences in $\mathrm{d} 13 \mathrm{C}$ and $\mathrm{d} 15 \mathrm{~N}$ values and $\mathrm{Hg}$ and $\mathrm{Cd}$ concentrations in cephalopods. Mar Ecol-Prog Ser 433:10720.

Commissione Europea, 2004. Regolamento del Parlamento Europeo e del Consiglio del 29 aprile 2004 relativo ai controlli ufficiali intesi a verificare la conformità alla normativa in materia di mangimi e di alimenti e alle norme sulla salute e sul benessere degli animali, 882/2004/CE. In: Gazzetta Ufficiale, L 165/1, 30/04/2004.

Commissione Europea, 2006. Regolamento del Parlamento Europeo e del Consiglio del 19 dicembre 2006 che definisce i tenori massimi di alcuni contaminanti nei prodotti alimentari, 1881/2006/CE. In: Gazzetta Ufficiale, L 364/5, 20/12/2006.

Commissione Europea, 2007. Regolamento del Parlamento Europeo e del Consiglio del 28 marzo 2007 relativo ai metodi di campionamento e di analisi per il controllo ufficiale dei tenori di piombo, cadmio, mercurio, stagno inorganico, 3-MCPD e benzo(a)pirene nei prodotti alimentari, 333/2007/CE. In: Gazzetta Ufficiale, L 88/29, 29/03/2007.
EFSA, 2009. Scientific opinion of the panel on contaminants in the food chain. Cadmium in food. EFSA Journal 980:1-139. Disponibile al sito: http://www.efsa.europa.eu/en/efsajournal/doc/980.pdf

EFSA, 2011. Scientific opinion of the panel on contaminants in the food chain. Statement on tolerable weekly intake for cadmium. EFSA Journal 9:1-19.

EFSA, 2012. Cadmium dietary exposure in the European population. EFSA Journal 10:2551. Disponibile al sito: http://www.efsa.europa.eu/it/efsajournal/d oc/2551.pdf

EPA, 1996. Method 3052. Microwave assisted acid digestion of siliceous and organically based matrices. Environmental Protection Agency ed., Boston, MA, USA.

EPA, 2007. Method 6020A. Inductively coupled plasma-mass spectrometry. Environmental Protection Agency ed., Boston, MA, USA.

ISO, 2005. Requisiti generali per la competenza dei laboratori di prova e taratura. Norma UNI EN ISO 17025/2005. Organizzazione internazionale per la normazione ed., Ginevra, Svizzera.

Manso M, Carvalho ML, Nunes ML, 2007. Characterization of essential and toxic elements in cephalopod tissues by EDXRF and AAS. X-Ray Spectrom 36:413-8.

Miramand P, Bustamante P, Bentley D, Kouéta $\mathrm{N}, 2006$. Variation of heavy metal concentrations ( $\mathrm{Ag}, \mathrm{Cd}, \mathrm{Co}, \mathrm{Cu}, \mathrm{Fe}, \mathrm{Pb}, \mathrm{V}$, and $\mathrm{Zn}$ ) during the life cycle of the common cuttlefish Sepia officinalis. Sci Total Environ 361:132-43.

Nessim R, Riad R, 2003. Bioaccumulation of heavy metals in Octopus vulgaris from coastal waters of Alexandria (Eastern Mediterranean). Chem Ecol 19:275-81.

Pastorelli AA, Morelli S, Baldini M, Stacchini P, Sagratella E, Zaza S, 2011. Valutazione dei livelli di presenza di cadmio in matrici alimentari: studio pilota a livello nazionale. J Food Sci Nutr 40:33-8.

Pereira P, Raimundo J, Vale C, Kadar E, 2009. Metal concentrations in digestive gland and mantle of Sepia officinalis from two coastal lagoons of Portugal. Sci Total Environ 407:1080-8.

Pierce GJ, Stowasser G, Hastie LC, Bustamante P, 2008. Geographic, seasonal and ontogenetic variation in cadmium and mercury concentrations in squid (Cephalopoda: Teuthoidea) from UK waters. Ecotox Environ Safe 70:422-32.

Pierce GJ, Allcock L, Bruno I, Bustamante P, González A, Guerra A, Jereb P, Lefkaditou E, Malham S, Moreno A, Pereira J, Piatkowski U, Rasero M, Sánchez P, Santos B, Santurtún M, Seixas S, Villanueva R, eds. 2010. Cephalopod biology and fisheries in Europe. ICES-Cooperative Research Report No. 303. International 
Council for the Exploration of the Sea ed., Copenhagen, Danimarca. Disponibile al sito: http://oceanrep.geomar.de/10074/1/ CRR-303\%2520final.pdf

Raimundo J, Caetano M, Vale C, 2004. Geographical variation and partition of metals in tissues of Octopus vulgaris along the Portuguese coast. Sci Total Environ 325:71-81.

Raimundo J, Costa PM, Vale C, Costa MH, Moura I, 2010a. Metallothioneins and trace elements in digestive gland, gills, kidney and gonads of Octopus vulgaris. Comp Biochem Phys C 152:139-46.

Raimundo J, Pereira P, Vale C, Caetano M, 2005. Fe, $\mathrm{Zn}, \mathrm{Cu}$ and $\mathrm{Cd}$ concentration in the digestive gland and muscle tissues of Octopus vulgaris and Sepia officinalis from two coastal areas in Portugal. Cienc Mar 31:243-51.

Raimundo J, Vale C, 2008. Partitioning of the $\mathrm{Fe}, \mathrm{Cu}, \mathrm{Zn}, \mathrm{Cd}$ and $\mathrm{Pb}$ concentrations among eleven tissue of Octopus vulgaris from the portuguese coast. Cienc Mar 34:297-305.

Raimundo J, Vale C, Duarte R, Moura I, 2008. Sub-cellular partitioning of $\mathrm{Zn}, \mathrm{Cu}, \mathrm{Cd}$ and $\mathrm{Pb}$ in the digestive gland of native Octopus vulgaris exposed to different metal concentrations (Portugal). Sci Total Environ 390:410-6.

Raimundo J, Vale C, Duarte R, Moura I, 2010b. Association of $\mathrm{Zn}, \mathrm{Cu}, \mathrm{Cd}$ and $\mathrm{Pb}$ with protein fractions and sub-cellular partitioning in the digestive gland of Octopus vulgaris living in habitats with different metal levels. Chemosphere 81:1314-9.

Seixas S, Bustamante P, Pierce GJ, 2005. Interannual patterns of variation in concentrations of trace elements in arms of Octopus vulgaris. Chemosphere 59:111324.

Storelli MM, Barone G, Marcotrigiano G0, 2005. Cadmium in cephalopod molluscs: implications for public health. J Food Protect 68:577-80.

Storelli MM, Garofalo R, Giungato D, Giacominelli-Stuffler R, 2010. Intake of essential and non-essential elements from consumption of octopus, cuttlefish and squid. Food Addit Contam B 3:14-8.

Storelli MM, Giacominelli-Stuffler R, Storelli A, Marcotrigiano G0, 2006. Cadmium and mercury in cephalopod molluscs: estimated weekly intake. Food Addit Contam 23:25-30.

Storelli MM, Marcotrigiano G0, 1999. Cadmium and total mercury in some cephalopods from the South Adriatic Sea (Italy). Food Addit Contam 16:261-5.

WHO, 2010. Preventing disease through health environments. Exposure to cadmium: a major public health concern. WHO ed., Ginevra, Svizzera. Disponibile al sito: http://www.who.int/ipcs/features/cadmium.pdf 